ANNALES

POLONICI MATHEMATICI

XII (1962)

\title{
A note on rational functions of several complex variables
}

\author{
by J. Siciak (Kraków)
}

Let $f(z)=f\left(z_{1}, \ldots, z_{n}\right), n \geqslant 2$, be holomorphic in a polycylinder $P=\left\{z:\left|z_{k}\right| \leqslant r, k=1, \ldots, n\right\}$. Then

$$
f(z)=\sum_{=0}^{\infty} P_{,}(z), \quad \text { for } \quad z \in P,
$$

where $P_{\nu}(z)=P_{v}\left(z_{1}, \ldots, z_{n}\right)$ is a homogeneous polynomial of degree $\nu$ and the series is uniformly convergent in $P$. Let

$$
g\left(t ; \theta_{2}, \ldots, \theta_{n}\right)=f\left(t, e^{i \theta_{2}} t, \ldots, e^{i \theta_{n}} t\right)=\sum_{i=0}^{\infty} P_{v}\left(1, e^{i \theta_{2}}, \ldots, e^{i \theta_{n}}\right) t^{\nu}
$$

and let $E_{k}, k=2,3, \ldots, n$, denote a subset of the interval $[0,2 \pi]$. The purpose of this note is to prove the following

Theorem. If $E_{i}, i=2,3, \ldots, n$, is non-denumerable and $g\left(t ; \theta_{2}, \ldots, \theta_{n}\right)$ is rational with respect to $t$ for any $\theta=\left(\theta_{2}, \ldots, \theta_{n}\right) \in E=E_{2} \times \ldots \times E_{n}$, then $f\left(z_{1}, \ldots, z_{n}\right)$ is rational with respect to $z=\left(z_{1}, \ldots, z_{n}\right)$. Moreover, if $E_{2}$ is at most denumerable and $E_{3}, \ldots, E_{n}$ are arbitrary, then there exists an entire transcendental function $f(z)$ such that $g\left(t ; \theta_{2}, \ldots, \theta_{n}\right)$ $=f\left(t, e^{i \theta_{2}} t, \ldots, e^{\theta i_{n}} t\right)$ is a polynomial in $t$ for each $\left(\theta_{2}, \ldots, \theta_{n}\right) \in E$.

Proof. The idea of the proof is based on Kronecker's necessary and sufficient condition for a function of one variable to be rational (see [1], p. 102-103).

Let $\theta=\left(\theta_{2}, \ldots, \theta_{n}\right)$ be a fixed point of $E$. The function $g(t ; \theta)$ $=g\left(t ; \theta_{2}, \ldots, \theta_{n}\right)$ can be written in the form

$$
g(t, \theta)=\frac{a_{0}+a_{1} t+\ldots+a_{k-1} t^{k-1}}{b_{0}+b_{1} t+\ldots+b_{k} t^{k}}
$$

where $a_{0}, a_{1}, \ldots, a_{k-1}, b_{0}, b_{1}, \ldots, b_{k}$ and $k$ depend on $\theta$. Since $g(t, \theta)$ $=\sum_{i=0}^{\infty} P_{v}\left(1, e^{i \theta_{2}}, \ldots, e^{i 0_{n}}\right) t^{\prime}$, we have

$$
a_{0}+a_{1} t+\ldots+a_{k-1} t^{k-1}=\left(P_{0}+P_{1} t+\ldots\right)\left(b_{0}+b_{1} t+\ldots+b_{k} t^{k}\right),
$$


whence

$$
\begin{aligned}
a_{0} & =b_{0} P_{0} \\
a_{1} & =b_{1} P_{0}+b_{0} P_{1}, \\
\cdot \cdot & \cdot \cdot \cdot \cdot \cdot \cdot \cdot \cdot \cdot \cdot \cdot \cdot \dot{\cdot} \cdot \cdot \cdot \\
a_{k-1} & =b_{k-1} P_{0}+b_{k-2} P_{1}+\ldots+b_{0} P_{k-1}, \\
0 & =b_{k} P_{0}+b_{k-1} P_{1}+\ldots+b_{0} P_{k}, \\
0 & =b_{k} P_{1}+b_{k-1} P_{2}+\ldots+b_{0} P_{k+1},
\end{aligned}
$$

Let

$$
\begin{aligned}
& C_{\lambda \mu}(z)=C_{\lambda \mu}\left(z_{1}, \ldots, z_{n}\right)
\end{aligned}
$$

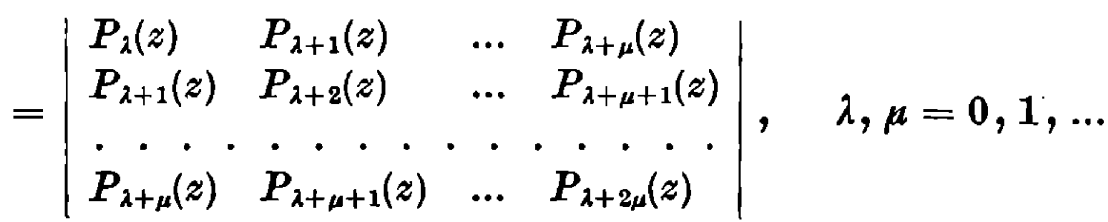

Since at least one of the coefficients $b_{0}, b_{1}, \ldots, b_{k}$ is different from zero, it follows from (4) that

$$
C_{\lambda \mu}\left(1, e^{i \theta_{2}}, \ldots, e^{i \theta_{n}}\right)=0, \quad \text { for } \quad \lambda \geqslant 0 \text { and } \mu=k=k(\theta) .
$$

Now we shall prove that there exist non-denumerable subsets $E_{i}^{0} \subset E_{i}$, $i=2,3, \ldots, n$, such that the function $k(\theta)=k_{0}=$ const for $\theta \in E^{0}=E_{2}^{0} \times$ $\times E_{8}^{0} \times \ldots \times E_{n}^{0}$. Indeed, let $\left(\theta_{2}^{0}, \theta_{9}^{0}, \ldots, \theta_{n-1}^{0}\right)$ be a fixed point of $E_{2} \times E_{3} \times$ $\times \ldots \times E_{n-1}$. Since the set of points $\left(\theta_{2}^{0}, \theta_{3}^{0}, \ldots, \theta_{n-1}^{0}, \theta_{n}\right)$, where $\theta_{n} \in E_{n}$, is non-denumerable and $k\left(\theta_{2}^{0}, \theta_{3}^{0}, \ldots, \theta_{n-1}^{0}, \theta_{n}\right)$ takes at most denumerably many values, there are a non-denumerable subset $E_{n}^{0}$ of $E_{n}$ and an integer $k_{1}\left(\theta_{2}^{0}, \theta_{3}^{0}, \ldots, \theta_{n-1}^{0}\right)$ such that $k\left(\theta_{2}^{0}, \theta_{3}^{0}, \ldots, \theta_{n-1}^{0}, \theta_{n}\right)=k_{1}\left(\theta_{2}^{0}, \theta_{3}^{0}, \ldots, \theta_{n-1}^{0}\right)$ for $\theta_{n} \in E_{n}^{0}$. Similarly, there is a non-denumerable subset $E_{n-1}^{0}$ of $E_{n-1}$, such that $k_{1}\left(\theta_{2}^{0}, \theta_{3}^{0}, \ldots, \theta_{n-2}^{0}, \theta_{n-1}\right)=k_{2}\left(\theta_{2}^{0}, \theta_{3}^{0}, \ldots, \theta_{n-2}^{0}\right)$ for $\theta_{n-1} \in E_{n-1}^{0}$. Therefore $k\left(\theta_{2}^{0}, \theta_{3}^{0}, \ldots, \theta_{n-2}^{0}, \theta_{n-1}, \theta_{n}\right)=k_{2}\left(\theta_{2}^{0}, \theta_{3}^{0}, \ldots, \theta_{n-2}^{0}\right)$ for $\left(\theta_{n-1}, \theta_{n}\right) \in E_{n-1}^{0} \times E_{n}^{0}$. By repeating the procedure, we shall find non-denamerable subsets $E_{n-k}^{0}$ of $E_{n-k}, k=0,1, \ldots, n-2$, and an integer $k_{0}$ such that

$$
k(\theta)=k\left(\theta_{2}, \ldots, \theta_{n}\right)=k_{0}, \quad \text { for } \quad \theta \in E^{0}=E_{2}^{0} \times E_{3}^{0} \times \ldots \times E_{n}^{0} .
$$

Therefore

$$
C_{\lambda \mu}\left(1, e^{i \theta_{2}}, \ldots, e^{i \theta_{n}}\right)=0 \quad \text { for } \quad \lambda \geqslant 0, \mu=k_{0}, \theta \in E^{0} .
$$

Since

we have

$$
P_{\lambda}\left(e^{i \varphi}, e^{i\left(\theta_{2}+\varphi\right)}, \ldots, e^{i\left(\theta_{n}+\varphi\right)}\right)=e^{i \lambda \phi} P_{\lambda}\left(1, e^{i \theta_{2}}, \ldots, e^{i \theta_{n}}\right)
$$

$$
C_{\lambda_{\mu}}\left(e^{i \varphi}, e^{i\left(\theta_{2}+\varphi\right)}, \ldots, e^{i\left(\theta_{n}+\varphi\right)}\right)=e^{i(\mu+1)(\lambda+\mu) \varphi} C_{\lambda \mu}\left(1, e^{i \theta_{2}}, \ldots, e^{i \theta_{n}}\right) .
$$

Thus, by (7), we have

$$
C_{\lambda \mu}\left(e^{i \varphi}, e^{i\left(\theta_{2}+\varphi\right)}, \ldots, t^{i\left(\theta_{n}+\varphi\right)}\right)=0,
$$


for $\lambda \geqslant 0, \mu=k_{0}, \theta \in E^{0}$ and $\varphi \in[0,2 \pi]$. Therefore the polynomial $C_{\lambda_{\mu}}\left(z_{1}, \ldots, z_{n}\right)$ vanishes identically if $\lambda \geqslant 0$ and $\mu=k_{0}$. At any point $z=\left(z_{1}, \ldots, z_{n}\right)$ the rank $r=r(z)$ of the matrix

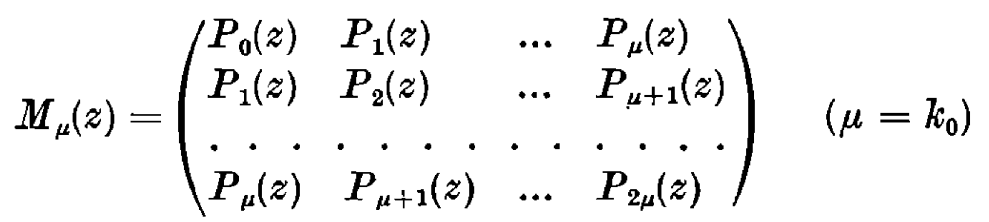

is less than or equal to $k_{0}, r \leqslant k_{0}$. Let $r_{0}=\max _{z \in C^{n}} r(z)=r\left(z^{0}\right)$ and let

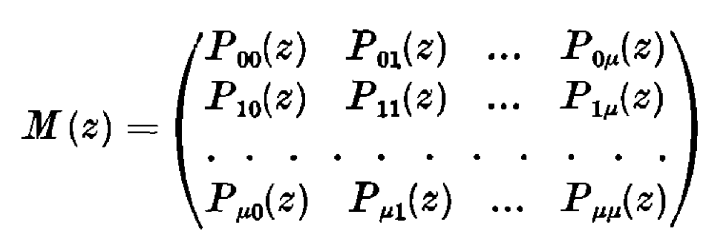

be a matrix which differs from $M_{\mu}(z)$ only by a permutation of its columns or rows. We choose the matrix $M(z)$ in such a way that

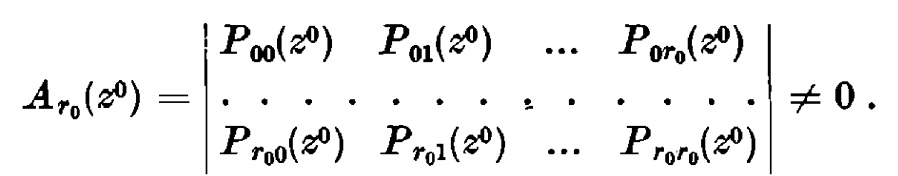

Therefore $A_{r_{0}}(z) \neq 0$ in a neighbourhood $U$ of $z^{0}$. Given $z \epsilon U$, consider the system of linear equations

(9) $P_{i 0}(z) e_{0}+P_{i 1}(z) e_{i}+\ldots+P_{i r_{0}}(z) e_{r_{0}}+\ldots+P_{i \mu}(z) e_{\mu}=0, \quad i=0,1, \ldots, \mu$, with respect to the unknowns $e_{i}, i=0,1, \ldots, \mu$. Putting $e_{r_{0}+1}=1$ and $e_{r_{0}+2}=e_{r_{0}+3}=\ldots=e_{\mu}=0$, we shall solve the system

(10) $P_{i 0}(z) e_{0}+P_{i 1}(z) e_{1}+\ldots+P_{i r_{0}}(z) e_{r_{0}}=-P_{i, r_{0}+1}(z), \quad i=0,1, \ldots, r_{0}$.

The solution $e_{0}(z), e_{1}(z), \ldots, e_{r_{0}}(z), e_{r_{0}+1}=1, e_{r_{0}+2}(z)=e_{r_{0}+9}(z)=\ldots=e_{\mu}(z)$ $\equiv 0$, is also a solution of system (9), and, moreover, the functions $e_{i}(z)$, $i=0, \ldots, \mu$ are rational with respect to $z=\left(z_{1}, \ldots, z_{n}\right)$ in the neighbourhood $U$. Therefore there exists a solution $b_{0}(z), \ldots, b_{\mu}(z)$ of the first $\mu$ equations of the infinite system

$$
\begin{array}{r}
P_{0}(z) b_{\mu}+P_{1}(z) b_{\mu-1}+\ldots+P_{\mu}(z) b_{0}=0 \\
P_{1}(z) b_{\mu}+P_{2}(z) b_{\mu-1}+\ldots+P_{\mu+1}(z) b_{0}=0
\end{array}
$$

and it is a proper permutation of the functions $e_{i}(z), i=0,1, \ldots, \mu$. Since $C_{\lambda \mu}(z)=0$ for $\lambda \geqslant 0, z \in C^{n}$, it follows that $b_{0}(z), b_{1}(z), \ldots, b_{\mu}(z)$ satisfy all the equations of (11). If we now put

$$
\begin{aligned}
& a_{0}(z)=b_{0}(z) P_{0}(z) \text {, } \\
& a_{1}(z)=b_{1}(z) P_{0}(z)+b_{0}(z) P_{1}(z) \\
& a_{\mu-1}(z)=b_{\mu-1}(z) P_{0}(z)+b_{\mu-2}(z) P_{1}(z)+\ldots+b_{0}(z) P_{\mu-1}(z),
\end{aligned}
$$


then $a_{0}(z), \ldots, a_{\mu-1}(z), b_{0}(z), \ldots, b_{\mu}(z)$ are rational and satisfy equations (11) and (12) for any $z \in C^{n}$. Therefore

$$
\sum_{p=0}^{\infty} P_{\nu}(z) t^{\prime}=\frac{a_{0}(z)+a_{1}(z) t+\ldots+a_{\mu-1}(z) t^{\mu-1}}{b_{0}(z)+b_{1}(z) t+\ldots+b_{\mu}(z) t^{\mu}} \quad\left(\mu=k_{0}\right)
$$

for $|t| \leqslant 2$ and $\left|z_{k}\right| \leqslant \varrho$, $\varrho$ being sufficiently small. We can check that

$$
\begin{aligned}
& \begin{aligned}
& a_{0}(z)+a_{1}(z) t+\ldots+a_{\mu-1}(z) t^{\mu-1} \\
& \quad=A_{0}(z)+A_{1}(z)(t-1)+\ldots+A_{\mu-1}(z)(t-1)^{\mu-1}, \\
& b_{0}(z)+b_{1}(z) t+\ldots+b_{\mu}(z) t^{\mu} \\
& \quad=B_{0}(z)+B_{1}(z)(t-1)+\ldots+B_{\mu}(z)(t-1)^{\mu}
\end{aligned}
\end{aligned}
$$

where $A_{i}(z), i=0, \ldots, \mu-1$, and $B_{k}(z), k=0,1, \ldots, \mu$, are polynomials in $a_{0}, \ldots, a_{\mu-1}$ and $b_{0}, \ldots, b_{\mu}$, respectively. (Thus $A_{i}(z)$ and $B_{k}(z)$ are rational functions in $z=\left(z_{1}, \ldots, z_{n}\right)$ ). Suppose that $B_{0}(z)=B_{1}(z)=\ldots$ $=B_{l-1}(z) \equiv 0$ and. $B_{l}(z) \not \equiv 0$. Such a $B_{l}(z)$ certainly exists because at least one of the $b_{0}(z), b_{1}(z), \ldots, b_{\mu}(z)$ does not vanish identically. Therefore, since $\lim _{t \rightarrow 1} \sum_{\nu=0}^{\infty} P_{\nu}(z) t^{\dagger}=\sum_{\nu=0}^{\infty} P_{\nu}(z)=f(z)$, we have by (13)

i.e. $f(z)$ is rational.

$$
f(z)=A_{l}(z) / B_{l}(z)
$$

To prove the second part of the theorem, let $E_{2}=\left\{e^{i \theta_{2}^{(v)}}\right\}, v=1,2, \ldots$, and put

$$
f\left(z_{1}, \ldots, z_{n}\right)=\sum_{\nu=1}^{\infty} \frac{1}{\nu !} \prod_{k=1}^{\infty}\left(z_{1}-e^{-\theta_{2}^{(k)}} z_{2}\right) .
$$

The function $f(z)$ is obviously entire and transcendental. Moreover,

$$
f\left(t, e^{i \theta_{2}^{(l)}} t, e^{i \theta_{3}} t, \ldots, e^{i \theta_{n}} t\right) \equiv \sum_{\nu=0}^{l-1} \frac{t^{v}}{v !} \prod_{k=1}^{j}\left(1-e^{i\left(\theta_{2}^{(n)}-\theta_{2}^{(k)}\right)}\right)
$$

for arbitrary $\theta_{3}, \theta_{4}, \ldots, \theta_{n}$.

CoRollakY. Weierstrass-Hurwitz's theorem ([2], p. 236). If a function $f(z)$ is meromorphic at any point $z \in C^{n}$ and at the point $(\infty, \infty, \ldots, \infty)$ (i.e. if $f\left(1 / z_{1}, 1 / z_{2}, \ldots, 1 / z_{n}\right)$ is meromorphic in a neighbourhood of $(0, \ldots, 0)$ ), then $f(z)$ is rational.

\section{References}

[1] G. Póly a und G. Szegö, Aufgaben und Lehrsätze II, Berlin 1925.

[2] Б. А. Фукс, Теория аналитических функиий многих комплексных переменных, Mосква 1948. 